OAI-PMH: http://www.indteca.com/ojs/index.php/Revista Scientific/oai

Artículo Original / Original Article

\title{
Acciones Pedagógicas para la Atención de Niños/as con Trastorno por Déficit de Atención e Hiperactividad (TDAH)
}

\author{
Autora: Andrea Karina Manrique Dávila \\ Universidad Pedagógica Experimental Libertador, UPEL \\ karikmanrique86@gmail.com \\ Barinas, Venezuela \\ https://orcid.org/0000-0003-1305-2646
}

Resumen

El presente artículo tuvo como propósito desarrollar acciones pedagógicas para la atención de niños/as con Trastorno por déficit de atención e hiperactividad (TDAH). Metodológicamente, se enmarcó en un paradigma cualitativo apoyado en una investigación acción a través de las fases: diagnóstico, planificación, ejecución y evaluación. Los informantes clave estuvieron conformados por (02) docentes de aula, un (01) especialista para recursos del aprendizaje. Para esto, fue aplicado la entrevista y dos instrumentos basados en el guion de entrevista y las notas de campo. Se concluyó que los entrevistados tienen escasez de formación en relación con el tema del Trastorno por déficit de atención e hiperactividad (TDAH). Situación que requiere diseñar actividades en un plan de acción para la atención de las diversas conductas manifestadas por los escolares, cuyos resultados generaron la existencia de serias dificultades en el abordaje de esta condición en los escolares, la cual permito la intervención con la presentación de charlas, estrategias y técnicas para el mejoramiento de la problemática.

Palabras clave: atención; formación; enseñanza; aprendizaje. 


\title{
Pedagogical Actions for the Care of Children with Disorder for Attention Deficiency and Hyperactivity (ADHD)
}

\begin{abstract}
The purpose of this article was to develop pedagogical actions for the attention of children with Attention Deficit Hyperactivity Disorder (ADHD). Methodologically, it was framed in a qualitative paradigm supported by an action research through the phases: diagnosis, planning, execution and evaluation. The key informants were made up of (02) classroom teachers, a (01) specialist for learning resources. For this, the interview was applied and two instruments based on the interview script and the field notes. It was concluded that the interviewees have a lack of training in relation to the issue of Attention Deficit Hyperactivity Disorder (ADHD). Situation that requires designing activities in a plan of action for the attention of the diverse behaviors manifested by the students, whose results generated the existence of serious difficulties in the approach of this condition in the students, which allowed the intervention with the presentation of talks, strategies and techniques for the improvement of the problem.
\end{abstract}

Keywords: attention; training; teaching; learning.

Date Received: 27-06-2018

Date Acceptance: 25-08-2018 


\section{Introducción}

En el contexto educativo, el trastorno por déficit de atención e hiperactividad (TDAH) contempla una variable multifactorial de suma preocupación para quienes se encargan del proceso de enseñanza aprendizaje, pues los síntomas de inquietud en las funciones propias del individuo provocan sin duda, numerosos rasgos conductuales que se contraponen con las normas y exigencias institucionales, ameritando con ello, un abordaje integral para reorientar sus estímulos inadecuados en favor de su progreso escolar. Sobre este particular Román, Peón, Galeano, Gómez y Martin (2008), plantea lo siguiente:

El Trastorno por Déficit de Atención e Hiperactividad (TDAH) es heterogéneo. Sus formas de manifestación son variables y se expresan en la evidente dificultad para concentrar la atención por mucho tiempo en una labor determinada y por las muestras de hiperactividad constante, ésta última manifestada clínicamente de tan variadas formas que lleva a los menores a cambiar de una tarea a otra sin acabar ninguna; todo lo cual, además de afectar la integración social y el rendimiento académico, constituye un verdadero reto para cualquier familia, debido al gran malestar y el deterioro significativo que provoca en el ámbito familiar, personal y/o social del niño (pág. 16).

En este sentido, la atención de los escolares con (TDAH), alberga en muchos casos situaciones contrarias, lo cual ponen entredicho su actuación en el aula, dado a sus innumerables comportamientos que agudizan el clima escolar. Para Liza, Gupta y Sagar (2013): "A pesar de la extensa literatura, para muchos docentes sigue siendo un problema desconocido, lo que dificulta el diagnóstico y por lo tanto retrasa las herramientas de tratamiento" (pág. 142).

Un aspecto importante en este tipo de anomalía, viene dado por una estilo inadecuado de manejo en el contexto cotidiano del individuo y marcado por un complejo diagnóstico desalentador de un profesional o psicólogo, 
psiquiatra o pediatra del desarrollo; situación, que por la condición de ser madre o padre, éstos desestiman la presencia de dicho trastorno.

Además, estos niños con sus formas de manifestación hiperactiva, quienes generalmente controlan y les aplican medidas inequívocas para orientar sus comportamientos, lejos de mejorar sus rasgos conductuales, etiquetan su figura como un individuo lleno de características desadaptadas. Esto genera inquietudes que hacen valer el desconocimiento del problema y por lo tanto, contribuye al deterioro del aprendizaje.

Según Flores e Iglesias, (2017): "El Trastorno por Déficit de Atención con Hiperactividad (TDAH) presenta una importante prevalencia en la población escolar. El alumnado con este trastorno encuentra dificultades para el aprendizaje, que conducen a un menor rendimiento educativo" (pág. 145). Por ello, los profesionales de la educación deben adoptar intervenciones pedagógicas dirigidas a poner en práctica un trabajo áulico que optimice las diversas áreas de funcionamiento social, académica, atencional y emocional a fin de contribuir con su desarrollo integral, y su vez, crear las condiciones necesarias para potenciar las capacidades creativas en la vida del sujeto y su contexto.

Indudablemente, el papel que juega el profesional docente abarca no solo la precisión de conocer las repuestas en los términos conductuales y cognitivos del escolar con (TDAH), sino configurar acciones pedagógicas que abran la posibilidad de mejorar sus comportamientos indeseados dentro del ambiente en que se desenvuelve. Además, de establecer un conjunto de ciertos criterios didácticos y metódicos que busquen un proceso de adaptación para que responda a las exigencias escolares a fin de suprimir el componente disociativo.

\subsection{Propósito de la investigación}

Desarrollar acciones pedagógicas dirigidas al docente de aula para el 
mejoramiento de la atención de niños/as con trastorno por déficit de atención e hiperactividad (TDAH) en la Unidad Educativa Dr. Ramón Reinoso Núñez durante el periodo escolar 2017-2018.

\section{Contexto temático de la situación problema}

En las instituciones educativas venezolanas, los niños con Trastorno por Déficit de Atención con Hiperactividad (TDAH), representa un conjunto humano con derechos consagrados en la Ley Orgánica de Educación del año 2009 , centrada en la idea de alcanzar un nuevo modelo fundamentado en la no exclusión de las escuelas. De manera que estos niños conviven y reciben formación educativa en instituciones regulares, la cual son atendidos por docentes de aula no especializados en el área.

Este panorama de atención escolar, comúnmente presenta dificultades en varios sentidos desde el propio quehacer docente, producto de múltiples deficiencias para configurar intervenciones pedagógicas que respondan de forma rigurosa y efectiva a los comportamientos manifestados por los escolares. Los docentes viven a diario la confrontación de retos, debido a las arduas condiciones en las que desarrollan su profesión, con ciertas limitaciones, con niños/as de diversos comportamientos, que se les espera conocerlos, comprenderlos y tratarlos con infinita paciencia y afecto.

Las intervenciones pedagógicas muestran indicadores pocos favorables para generar resultados positivos en los términos de progreso escolar, por lo cual el profesional docente según Aparicio (2013): "Deben tomar conciencia de los procesos cognitivos que se utilizan en el aprendizaje para aprender a controlarlos, reconducirlos y optimizarlos" (pág. 41). Además, de extender escenarios significativos para reorientar sus estímulos que no excedan su capacidad de atención.

Debido a la complejidad de la labor docente; esta debe resolverse con la colaboración de todas las partes implicadas, familia, escuela y comunidad 
para lograr de manera conjunto el éxito de esta condición individual del escolar con (TDAH). Por tanto, el tratamiento de esta respuesta conductual debe valerse de condiciones enmarcadas en la paciencia, tolerancia y flexibilidad como un elemento clave para precisar con profundidad los niveles de comportamiento indeseados y formular tareas de atención pedagógica.

Una de las conclusiones más destacadas desde la propia gestión del docente para abordar este tipo de conducta en la vida del escolar, es la manera en que se organiza y plantea las metodologías de aprendizaje, la cual en muchas ocasiones manifiestan desigualdades por el carácter integral que tiene para ser aplicado de forma individual en el aula escolar. Esto se afirma también con lo que expresa Korzeniowsk y Ison (2008): argumenta lo siguiente:

Los docentes usualmente no disponen de los recursos necesarios para abordar en el aula los síntomas del TDAH. Generalmente, intentan corregirlos con restricciones, castigos y llamadas de atención en público. Sin embargo, estos métodos no resultan exitosos y su uso frecuente suele ocasionar nuevos inconvenientes. El alumno suele ser rotulado como el niño problema del grupo, lo que agudiza aún más sus dificultades académicas, emocionales e interpersonales (pág. 66).

En la actualidad diversas investigaciones en el ámbito escolar, según Jarque, Tárraga y Miranda (2007), citados en Frutos, Gutiérrez y Ortuño, (2014): vinculado a "Ios conocimientos del profesorado en torno al TDAH pone de manifiesto que en general, la formación en este ámbito no parece ser suficiente para atender adecuadamente las necesidades de estos alumnos y que es preciso que aumenten tales conocimientos" (pág. 3).

Ciertamente, son diversas las variables presenten el proceso de atención del niño/a con (TDAH), en la cual el docente puede convertirse en el eje clave para abrir un campo pedagógico cargado de líneas de acción que facilite un diagnóstico, tratamiento y una atención conjunta de solución efectiva ante los variados comportamientos que se expresan en el contexto académico. 
Para ello, desde la actuación docente el abordaje de este trastorno debe precisar, según la opinión de Martínez, Gutiérrez y Ortuño, (2014): "saber que entre los ajustes a realizar no se contempla el aprobarle todo o bajar el nivel de exigencia académica, sino adaptar las tareas y deberes a sus peculiaridades" (pág. 71).

De igual forma se apunta que los síntomas de inatención e hiperactividad pueden provocar rupturas en el progreso escolar, sino se llega a focalizar un adecuado diagnóstico y planear acciones pedagógicas que acompañe la reorientación de la problemática conductual en el trabajo de clase, es decir, albergue los materiales didácticos, las tareas pedagogías, la organización del ambiente, el comportamiento del grupo y la participación de los implicados como partes esenciales para ayudar a maximizar las potencialidades del escolar con esta condición anómala.

En el marco de estas consideraciones, es evidente entonces, la necesidad de profundizar sobre las deficiencias pedagógicas que afectan el abordaje de este trastorno; por ello, se tiene como finalidad desarrollar acciones pedagógicas dirigidas al docente de aula para la atención a niños(as) con trastorno por déficit de atención e hiperactividad (TDAH) en la Unidad Educativa Dr. Ramón Reinoso Núñez. Por lo antes mencionado, se plantea dar respuesta a las siguientes formulaciones de investigación:

¿Qué acciones pedagógicas utilizan los docentes de aula de la U.E. Dr. Ramón Reinoso Núñez para el mejoramiento de esta patología conductual? ¿Cuáles acciones pedagógicas dirigidas a los docentes de aula se pueden planificar para el mejoramiento de la conducta inapropiada por este grupo escolar? ¿Cómo sería la ejecución de las acciones pedagógicas dirigidas a los docentes en la U.E. Dr. Ramón Reinoso Núñez, para el mejoramiento de la atención de niños/as que presenta TDAH? ¿Cómo se llevaría a cabo la evaluación de las acciones pedagógicas utilizadas por los docentes para el mejoramiento de la atención con niños y niñas que presentan TDAH? 


\section{Referentes Teóricos}

\subsection{Teoría Cognitivo Social de Bandura}

En la presente investigación las teorías se direccionan hacia la ubicación de la profundidad de la variable de estudio definida en función de acciones pedagógicas dirigidas al docente de aula para el mejoramiento de la atención de niños/as con trastorno por déficit de atención e hiperactividad (TDAH).

De allí que hacer referencia a la conducta del ser humano es un aspecto complejo, la cual está caracterizado por una serie de explicaciones en distintas vertientes, entre las que destacan las posiciones conductistas y las posiciones cognoscitivistas e incluso se presentan autores que toman postulados de unas y otras referidas a la Teoría Social de Albert Bandura, quien se considera uno de los más importantes representantes del Cognoscitivismo. En esta línea, la Teoría Social, Schunk (1997), sustenta lo siguiente:

Bandura analiza la conducta humana dentro del marco teórico de la reciprocidad triádica, las interacciones recíprocas de conductas, variables ambientales y factores personales como las cogniciones... Según la postura cognoscitiva social, la gente no es impulsada por fuerzas internas ni controlada y moldeada automáticamente por estímulos externos. No, el funcionamiento humano se explica en términos de un modelo de reciprocidad triádica... (pág. 108).

Para Bandura, existen elementos modeladores de la conducta del individuo, que actúan como sinergias para la generación de la respuesta humana, y en ese sentido la mente analiza el contexto inmediato concediendo un significado observacional a las experiencias para luego manifestar patrones conductuales que le hacen comprender su contexto.

De esta forma en el ambiente educativo, el ser humano logra un aprendizaje en la medida en que alcanza a asimilar y comprender la realidad adaptando modelos que le presenta el medio, por lo tanto, la conducta es el 
resultado de las deducciones, análisis y pensamientos del individuo hacia todos los elementos del medio, desarrollando habilidades, capacidades y competencias en cada uno.

\subsection{Trastorno por déficit de atención e hiperactividad (TDAH)}

En la vida de individuo con (TDAH), es una de las complejas anomalías responsables de respuestas indeseadas asociados básicamente a la atención y de comportamientos para conservar niveles de atención durante un prolongado tiempo determinado. Para Valenzuela y Gómez (2010): “El Trastorno por Déficit de Atención e Hiperactividad (TDAH) es definido como un síndrome conductual de origen neurobiológico, caracterizado por síntomas manifiestos de desatención y/o impulsividad-hiperactividad" (pág. 23).

Además, existen respuestas comunes que se relacionan al observar las personas con relación a este patrón conductual, las cuales se ven reflejadas por las diversas dificultades que compromete su funcionamiento cotidiano, reseñadas por Orjales (2005):

Dificultad de recuperar en su memoria aquello aprendido con anterioridad para aplicarlo a su momento actual, Dificultad en la percepción y control del tiempo, Pobres habilidades de organización de la conducta en el tiempo, Dificultad en hacer referencias a hechos del pasado o del futuro y alteraciones en la utilización de conceptos temporales (pág. 86).

Sobre esto, se deduce que los niños/as con esta anomalía manifiestan una conducta limitada para desarrollar plenamente la percepción y retención en la memoria de hechos o situaciones temporales, que lo conducen a una restringida participación en los procesos académicos y a su vez, interponen serias barreras para adquirir un aprendizaje significativo junto al resto de su grupo escolar. 


\section{Marco Metodológico}

Dentro de este marco, es importante señalar que la estructura metodológica del estudio está circunscrita en la investigación acción participativa, por lo que Petit, Copello y Bianchi (2015): reseñan que "El método de investigación acción participativa es el indicado cuando el investigador se propone no únicamente conocer la realidad específica de un grupo, sino que además desea actuar" (pág. 172).

De forma que a partir de esta modalidad los actores sociales investigados activan su figura como rol de coinvestigadores en cada una de las etapas de procesos de indagación y transformación de su contexto, por cuanto en el transito van adquiriendo patrones de comportamiento asociados a la concienciación, desarrollo y emancipación con respecto a su evento problematizado. De allí que el estudio basado en investigación acción centra su objetivo en desarrollar un plan de acciones pedagógicas dirigidas al docente de aula para el mejoramiento de la atención de niños/as con trastorno por déficit de atención e hiperactividad (TDAH) en la Unidad Educativa Dr. Ramón Reinoso Núñez durante el periodo escolar 2017-2018.

La investigación acción participante genera el conocimiento bajo una metodología que tomó en cuenta el planteamiento de Hurtado y Toro (2001), quienes dividen "el proceso de trabajo de una investigación acción participante en cuatro etapas: diagnóstico, planificación, ejecución y evaluación" (pág. 120).

Fase I: Diagnóstico: En la investigación acción participación, el diagnostico contempla la primera fase de la espiral de la intervención, y se refiere a la precisión de la temática o problema del contexto social. Latorre (2007a): afirma que en esta etapa "Es preciso hacer un reconocimiento o diagnóstico del problema encontrado, la finalidad es hacer una descripción y explicación comprensiva de la situación actual, obtener evidencias que sirvan de punto de partida para desarrollar la investigación" (pág. 47). 
Fase II Planificación: Esta fase se caracteriza por presentar las acciones que dan solución al problema descrito, el cual está compuesto por una serie de actividades que serán desarrolladas para solventar la realidad existente. Santos, Díaz y Lautín (2011), afirma que esta fase:

Está integrada por el sistema de acciones, tareas y/o indicadores propuestos para alcanzar los resultados esperados y el cumplimiento del objetivo. Estas acciones, tareas y/o indicadores presentan un carácter operativo o educativo, cuantitativo o cualitativo; según se relacionen con las evaluaciones de los diferentes momentos de la investigación (pág. 85).

En tal sentido, esta fase se lleva a cabo a través de un plan de acción con los siguientes elementos: finalidades, actividades, responsable(s), duración, estrategia y la evaluación, tomando en cuenta también el tiempo disponible, recursos humanos y económicos, así como las fechas de ejecución y la evaluación de las acciones.

Fase III Ejecución: Representa la fase que permite poner en práctica las acciones planificadas, describiéndose cada uno de los sucesos ocurridos, cuyo fin es reflejar los resultados de la planificación, procesarlos e interpretarlos, utilizando los recursos previstos y cumpliendo con exactitud las acciones planificadas por medio de su aplicación en el contexto. Latorre (2007b): expresa "pone en marcha el trabajo investigativo propiamente dicho, pues consiste en la aplicación de las actividades y estrategias (...) previstas en el proyecto" (pág. 175).

Fase IV Evaluación: Se caracteriza porque le permite al investigador evaluar los resultados, haciendo las correcciones necesarias del proceso, analizar los logros alcanzados y hacer la negociación respectiva de acuerdo con los resultados obtenidos, mejorando y enriqueciendo lo ejecutado, para tener la posibilidad de establecer nuevas metas. Latorre (2007c): afirma que

Los datos contienen pruebas o evidencias sobre las acciones 
que el investigador puede utilizar como particular interpretación y explicación de la acción. Cada vez que se completa un ciclo de investigación acción se disponen de registros de control y evaluación del ciclo. Estos registros son los datos que se puede utilizar para mostrar las mejoras que han tenido lugar (pág. 50).

En este sentido, esta etapa se considera importante dentro del estudio, porque se realiza el análisis de los hechos con base a los resultados que se obtuvieron a partir de las acciones ejecutadas, igualmente se lleva a cabo el análisis para obtener las conclusiones con la intención de mejorar las futuras acciones.

\subsection{Enfoque de la investigación}

La educación es un campo de estudio sobre la cual, se abordan y se tratan problemas educativos, por esto, es importante considerar la aplicación de metodologías pertinentes que generen nuevos conocimientos. Por esta razón, se inscribe dentro de la investigación de campo, la cual es vista como una acción de análisis sistemático que realiza el investigador en la realidad donde ocurre el evento con la intención de comprender su naturaleza. De acuerdo con esto, Palella y Martins (2010), expresan:

La Investigación de campo consiste en la recolección de datos directamente de la realidad donde ocurren los hechos, sin manipular o controlar las variables. Estudia los fenómenos sociales en su ambiente natural. El investigador no manipula variables debido a que esto hace perder el ambiente de naturalidad en el cual se manifiesta (pág. 88).

En este sentido, la investigación de campo el investigador posibilita herramientas participantes para entrar en contacto con el fenómeno de estudio, y encontrar el sentido de las subjetividades y significados cotidianos que poseen los informantes a fin de enriquecer la descripción contextual de lo que se investiga. 


\subsection{El contexto y los informantes}

Para entrar en contacto con el escenario de investigación, se hizo necesaria la selección de informantes pertenecientes a este grupo social, a fines de ubicar información de interés en la conformación del corpus del estudio. Sobre este particular, Hernández, Fernández y Baptista (2010a): señala que los informantes "es un método antropológico estándar ampliamente usado en las investigaciones y este término se aplica a cualquier persona que pueda brindar información detallada debido a su experiencia o conocimiento de un tema específico" (pág. 21).

De allí que, los informantes de la presente investigación se delimitaron en el ámbito del Grupo Escolar Estatal "Dr. Ramón Reinoso Núñez, por lo que se tomaron en cuenta 3 informantes, dos (02) docentes de aula, un (01) especialista para recursos del aprendizaje.

\subsection{Recolección de las evidencias}

Al respecto, la recolección de las evidencias es definida por Hernández, Fernández y Baptista (2010b): como aquella que "permite recolectar datos pertinentes sobre variables, sucesos, contextos, categorías, comunidades $u$ objetos, involucrados en la investigación" (pág. 244). De conformidad con esto, las técnicas utilizadas para la investigación fue la observación participante, determinada por Victorelli, Almeida, Dos Santos, Garcia, Ribeiro, y Mendes (2014): "es una estrategia de recogida de la información caracterizada por interacciones sociales profundas entre el investigador y el investigado que ocurren en el ambiente de estos" (pág. 76).

Por su parte, otra técnica empleada en la investigación es la entrevista vista como medio de apoyo para entrar en contacto directo con los informantes a fin de aprehender la información del fenómeno en su ambiente natural, al respecto, Martín (2005), expone que:

La entrevista es un proceso comunicativo en el cual la 
interacción de, al menos, un sujeto y un entrevistador intenta recuperar la percepción, el significado de un determinado objeto de estudio mediante la reconstrucción de los procesos, los contenidos y los significados de las experiencias personales del sujeto entrevistado (pág. 171).

Con base a lo planteado, a través de la entrevista se extrae información tacita sobre el evento de estudio, detallándose en aquellos aspectos de interés cualitativo del acontecimiento, relato, sentimientos que son imposibles captarse por medio de la observación.

Por otro lado, y con relación a los instrumentos para el registro de la información, se elaboró un guion, la cual según Valles (2007): "se caracteriza por la preparación de un guion de temas a tratar y por tener libertad el entrevistador para ordenar y formular las preguntas, a lo largo del encuentro de entrevista" (pág. 180). Los guiones utilizados se elaboraron a partir de las principales unidades de análisis de la investigación, aspecto que permitió establecer la interacción del investigador con los informantes clave.

En el mismo orden de ideas, se usó las notas de campo como el instrumento para recoger las anotaciones derivadas de la observación del fenómeno, definido por Monistrol (2007): "el instrumento de registros de datos del investigador de campo, donde se anotan las observaciones (notas de campo) de forma completa, precisa y detallada" (pág. 3). De acuerdo con esto, es una herramienta que permitió a la investigadora sistematizar las experiencias observadas en el desarrollo de las acciones para luego analizar los resultados y contrastar con las teorías relacionadas que sustentan el estudio investigativo.

\subsection{Validación y fiabilidad de los hallazgos}

En los estudios cualitativos la validez representa sin duda un requerimiento formal para lograr el rigor científico de los datos y con ello, 
establecer criterios de credibilidad en la investigación. Para el caso del presente estudio se utilizó la triangulación de datos, según Villamar (2015): es entendida como la "técnica de confrontación y herramienta de comparación de diferentes tipos de análisis de datos de un mismo objeto con la finalidad de contribuir a validar entrevistas, análisis documentales y hallazgos encontrados" (pág. 65).

Bajo esta premisa la triangulación de la información se realizó mediante las observaciones recopiladas por la investigadora en el docente y los estudiantes de 6to año sección "A", en la que se determinó una estrecha relación entre las entrevistas, las observaciones y los presupuestos encontrados en el aula.

Por su parte, la fiabilidad en las investigaciones cualitativas es un criterio importante para brindarle rigurosidad metodológica a los datos recopilados en el escenario de investigación. Bracker (2000): expresa que "La fiabilidad hace referencia a la posibilidad de replicar los estudios. Es decir, que un investigador que utilice los mismos métodos que otro llegue a los mismos resultados" (pág. 65).

Para lograr lo anterior, se empleó la fiabilidad sincrónica como una técnica para representar las semejanzas de las observaciones en el mismo periodo de tiempo en que se recolectaron, así lo expresa Arriagada, Burgos y Novoa (2008):

La fiabilidad sincrónica es la que da a conocer semejanzas en las observaciones realizadas dentro del mismo tiempo, esto no quiere decir que sean idénticas, sino que los hechos son relevantes y mencionan ciertas igualdades, pero también poseen diferencias características de cada una de ellas (pág. 119).

Con base a esto, en la consistencia o fiabilidad de los instrumentos aplicados, se comprende sus resultados con la intención de que los mismos sean similares en cuanto a su información, de manera que en el estudio la 
fiabilidad fue encontrada al momento de comparar los instrumentos, donde se precisó su alto grado de fiabilidad, arrojando debilidades con relación al abordaje pedagógico de las conductas manifestadas.

\subsection{Análisis la información}

En el tratamiento de la información es indispensable emplear un conjunto de técnicas para captar el sentido de los datos, de allí Quecedo y Castaño (2002): señalan que "el análisis como un proceso de pensamiento que implica el examen sistemático de algo para determinar sus partes, las relaciones entre las partes, y sus relaciones con el todo" (pág. 5).

De acuerdo con esto, en las investigaciones cualitativas por su flexibilidad en el diseño, se organizó la información siguiendo lo plasmado en la triangulación metodológica, es decir, la vinculación existente que se produjeron en el análisis de cada constructo respondido por los informantes clave tales como el docente, los estudiantes y la investigadora encargada del estudio.

\section{Consideraciones Finales}

Luego de realizar el diagnostico con los instrumentos y recursos de investigación, se demostró que hay una necesidad sustancial que le otorgan significatividad al desarrollo de acciones pedagógicas dirigidas al docente de aula para la atención a niños(as) con trastorno por déficit de atención e hiperactividad (TDAH) en la Unidad Educativa Dr. Ramón Reinoso Núñez, porque los docentes ameritan a un cambio o transposición didáctica en su quehacer pedagógico que contribuya a la reflexión y a la creación de un nueva manera de generar posibilidades didácticas para mejorar el abordaje de ese trastorno en los escolares.

Los docentes poseen dificultades para emprender el trabajo o formas didácticas para atender esta anomalía, lo cual permite establecer escenarios 
que imposibilitan la concreción de acciones para atender de manera integral los comportamientos o conductas indeseadas en estos grupos escolares caracterizados por esta anomalía.

Asimismo, las acciones pedagógicas están centradas en la esencia curricular, por lo que los síntomas de inatención, hiperactividad e impulsividad adquieren ritmos de intensidad de carácter desadaptados que perturban la armonía del proceso de enseñanza aprendizaje en el aula escolar.

Del mismo modo, los maestros a pesar de jugar un papel preponderante en la intervención de este grupo escolar; los aspectos en términos de conocimientos, destrezas, habilidades y técnicas para manejar los rasgos de conductas presentan serias dificultares a la hora de su puesta en práctica, lo cuales crean conflictos en las tareas escolares que se concretan en el aula.

En las intervenciones pedagógicas se desarrollaron charlas de sensibilización, construcción de estrategias, técnicas y recursos didácticos basados en mecanismos cognitivos conductuales con la participación de todos los involucrados, en la que se aprendieron formas de abordar la reflexibilidad, intolerancia y alteraciones de las impulsividades. Finalmente, se llevó a cabo evaluaciones de las acciones, haciendo énfasis en sus alcances y su efectividad en la institución a objeto de estudio.

\section{Referencias}

Aparicio, F., (2013). Orientaciones y estrategias dirigidas al profesorado para trabajar con alumnado con trastorno por déficit de atención e hiperactividad. España: Materiales de apoyo a la acción educativa.

Arriagada, C., Burgos, Ú., \& Novoa, P. (2008). Catastro en el nivel de Educación Parvularia en la Comuna de Chillán. Seminario para optar al título de educadora de párvulos. Chillán, Chile: Universidad de Bío-Bío. Recuperado de:

http://repobib.ubiobio.cl/jspui/bitstream/123456789/171/1/Burgos Gon 


\section{z\%C3\%A1lez \%C3\%9Arsula.pdf}

Bracker, M, (2000). Metodología de la investigación cualitativa. Nicaragua: Sinopsis.

Flores, J., \& Iglesias, A. (2017). Conocimientos sobre el trastorno por déficit de atención con hiperactividad (TDAH) en futuros profesionales del ámbito escolar. Bordón. Revista de Pedagogía, 69(3), 145-149. ISSN: 2340-6577. Recuperado de:

https://doi.org/10.13042/Bordon.2017.50278

Frutos, M., Gutiérrez, E., \& Ortuño, J. (2014). Conocimientos y lagunas de Ios docentes sobre el TDAH: la importancia de la formación. Murcia, España: Consejería de educación, Cultura y Universidades.

Hernández, S., Fernández, C., \& Baptista, L. (2010a,b). Metodología de la Investigación. México: McGraw-Hill.

Hurtado, I. \& Toro, J. (2007). Paradigmas y Métodos de la Investigación en Tiempos de Cambio. Venezuela: Editorial El Nacional.

Korzeniowsk, C., \& Ison, M. (2008). Estrategias psicoeducativas para padres y docentes de niños con TDAH. Revista Argentina de Clínica Psicológica, XVII(1), 65-71. Recuperado de:

http://www.redalyc.org/articulo.oa?id=281921796006

Latorre, A. (2007a,b,c). La Investigación-Acción Conocer y Cambiar la Práctica Educativa. Barcelona: España: Editorial Graó.

Liza, D., Gupta, R., \& Sagar, R. (2013). Effect of educational module on knowledge of primaryschool teachers regarding early symptoms of childhood psychiatric disorders. Indian Journal of Psychological Medicine, 35(4), 368-72. Recuperado de: https://doi.org/10.4103/0253$\underline{7176.122228}$

Martín, M. (2005). Violencia juvenil exogrupal. Hacia la construcción de un modelo casual. Madrid, España: Secretaria General Técnica.

Martínez, M., Gutiérrez, E., \& Ortuño, J. (2014). Conocimientos y lagunas de 
los docentes sobre el TDAH: la importancia de la formación. En: Navarro, J.; Gracia, M.; Lineros, R.; y Soto, F. (Coords.). Claves para una educación diversa. Murcia, España: Consejería de Educación, Cultura y Universidades.

Monistrol, O. (2007). El trabajo de campo en investigación cualitativa. Nure investigación, (29), 1-4, ISSN: 1697-218X. Recuperado de:

http://www.nureinvestigacion.es/OJS/index.php/nure/article/view/339

Orjales, I. (2005). Déficit de atención con hiperactividad. Manual para padres y Educadores. Madrid, España: CEPE.

Palella, S., \& Martins, F. (2010). Metodología de la investigación cuantitativa. Caracas, Venezuela: FEDUPEL.

Petit, L., \& Copello, M., \& Bianchi, Y. (2015). Reflexiones en torno a las prácticas educativas del Diploma de Operador Socioeducativo en Economía Social y Solidaria en clave de Investigación Acción Participativa. + E / Revista de Extensión Universitaria, (5), 168-173. Recuperado de:

https://bibliotecavirtual.unl.edu.ar/ojs/index.php/Extension/article/view/ $\underline{5157}$

Quecedo, R., \& Castaño, C. (2002). Introducción a la metodología de investigación cualitativa. Revista de Psicodidáctica, (14), 5-39, ISSN: 1136-1034. Recuperado de:

http://www.redalyc.org/articulo.oa?id=17501402

Román, M., Peón, A., Galiano, C., Gómez, M., \& Martín., M. (2008). Normas de procedimientos diagnósticos y terapéuticos. Trastorno por Déficit de Atención con /sin Hiperactividad. Habana, Cuba: Ministerio de Salud Pública.

Santos, H., Díaz, F., \& Lautín, I. (2011). La Investigación Acción Participativa: posibilidades de aplicación en el contexto actual de Cuba. Luz, 10(2), 78-87. ISSN: 1814-151X. Recuperado de: 
http://luz.uho.edu.cu/index.php/luz/article/view/519

Schunk, D. (1997). Teorías del aprendizaje. México: Editorial Pearson Educación.

Valenzuela, A., \& Gómez, R. (2010). Trastorno por Déficit de Atención e Hiperactividad. Revista médica MD, 1.2(1), 23-27. Recuperado de:

http://www.medigraphic.com/cgi-

bin/new/resumen.cgi?IDARTICULO=39046

Valles, M. (2007). Entrevistas cualitativas. Madrid, España: CIS.

Victorelli, K., Almeida, A., Dos Santos, C., Garcia, C., Ribeiro, P., \& Mendes, M. (2014). Hablando de la Observación Participante en la investigación cualitativa en el proceso salud-enfermedad. Index de enfermería, 23(1-2), 75-79, ISSN: 1132-1296. Recuperado de:

http://www.index-f.com/index-enfermeria/v23n1-2/9228r.php

Villamar, M. (2015), La triangulación en la investigación científica como soporte al plan integral para estandarizar gastos logísticos generados por el proceso de transporte internacional de mercancías. Yachana, 4, 65-71, ISSN: 1390-7778. Recuperado de: http://revistas.ulvr.edu.ec/index.php/yachana/article/view/81/71 


\section{Andrea Karina Manrique Dávila \\ e-mail: karikmanrique86@gmail.com}

Nacida en Venezuela, Licencia en Educación mención

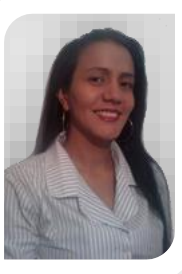
Integral (UNELLEZ, 2007), Docente para el Ministerio del Poder Popular para la Educación, cursos realizados adquisición y desarrollo del lenguaje, currículo, edumática, multimedia, didáctica de procesos del aprendizaje en el escolar, introducción a la filosofía, introducción a los medios de comunicación, evaluación escolar en el proyecto de aprendizaje, taller de alternativas metodológicas para la facilitación en el área de ciencias, participó como ponente en actividades de investigación científica.

El contenido de este manuscrito se difunde bajo una Licencia de Creative Commons ReconocimientoNoComercial-Compartirlgual 4.0 Internacional 